\title{
DISPOSITIF POUR L'ÉTUDE EXPÉRIMENTALE DE LA VARIATION AVEC LA TEMPÉRATURE DE LA FRÉQUENCE DE RÉSONANCE QUADRIPOLAIRE PURE DE L'AZOTE. APPLICATION A L'ORTHOPHÉNYLẼNEDIAMINE ET A LA 2-PICOLINE
}

\author{
par G. MALLET, L. GUIBE et D. VASILESCU
}

Laboratoire de Biophysique, Faculté des Sciences, Université de Nice. Institut d'Electronique Fondamentale, Faculté des Sciences d'Orsay

(Reçu le 30 novembre 1970)

\begin{abstract}
Résumé. - Nous décrivons un montage réalisé pour l'étude de l'influence de la température sur les fréquences de résonance quadripolaire pure de l'azote (entre $-196^{\circ} \mathrm{C}$ et $97^{\circ} \mathrm{C}$ ). Nous donnons une interprétation des couplages quadripolaires observés à $-196^{\circ} \mathrm{C}$ pour l'orthophénylènediamine, la 2-picoline et le cyanure d'allyle, ainsi que les résultats d'une étude en fonction de la température pour les deux premiers corps. Une transition de phase a été observée vers $-130^{\circ} \mathrm{C}$ dans la 2-picoline.
\end{abstract}

Abstract. - An experimental set for the determination of the temperature dependance of nitrogen pure quadrupole resonance lines (between $-196^{\circ} \mathrm{Cet} 97^{\circ} \mathrm{C}$ ) is described. The quadrupole couplings, measured at $-196^{\circ} \mathrm{C}$, are reported for orthophenylenediamine, 2-picoline and allylcyanide and tentative interpretation are given. The temperature dependance of the resonances has been studied for the two first compounds and a phase transition has been detected at $-130^{\circ} \mathrm{C}$ in 2-picoline.

1. Dispositif expérimental. - 1.1 LE SPECTROGRAPHE. - Le spectrographe réalisé [1] à Nice est très semblable à celui utilisé par l'un de nous à Orsay [2]. Nous ne considérons que la section haute fréquence, les circuits basse fréquence étant tout à fait classiques. Le montage original fonctionnant de façon satisfai- sante, nous ne l'avons pas modifié, bien que l'emploi des semi-conducteurs puisse paraître souhaitable pour réduire des dérives thermiques, des sources de vieillissement, des montages à lampes, et pour éliminer des résonances parasites attribuables aux lampes.

Le montage (Fig. 1) est du type Robinson [3], [4]

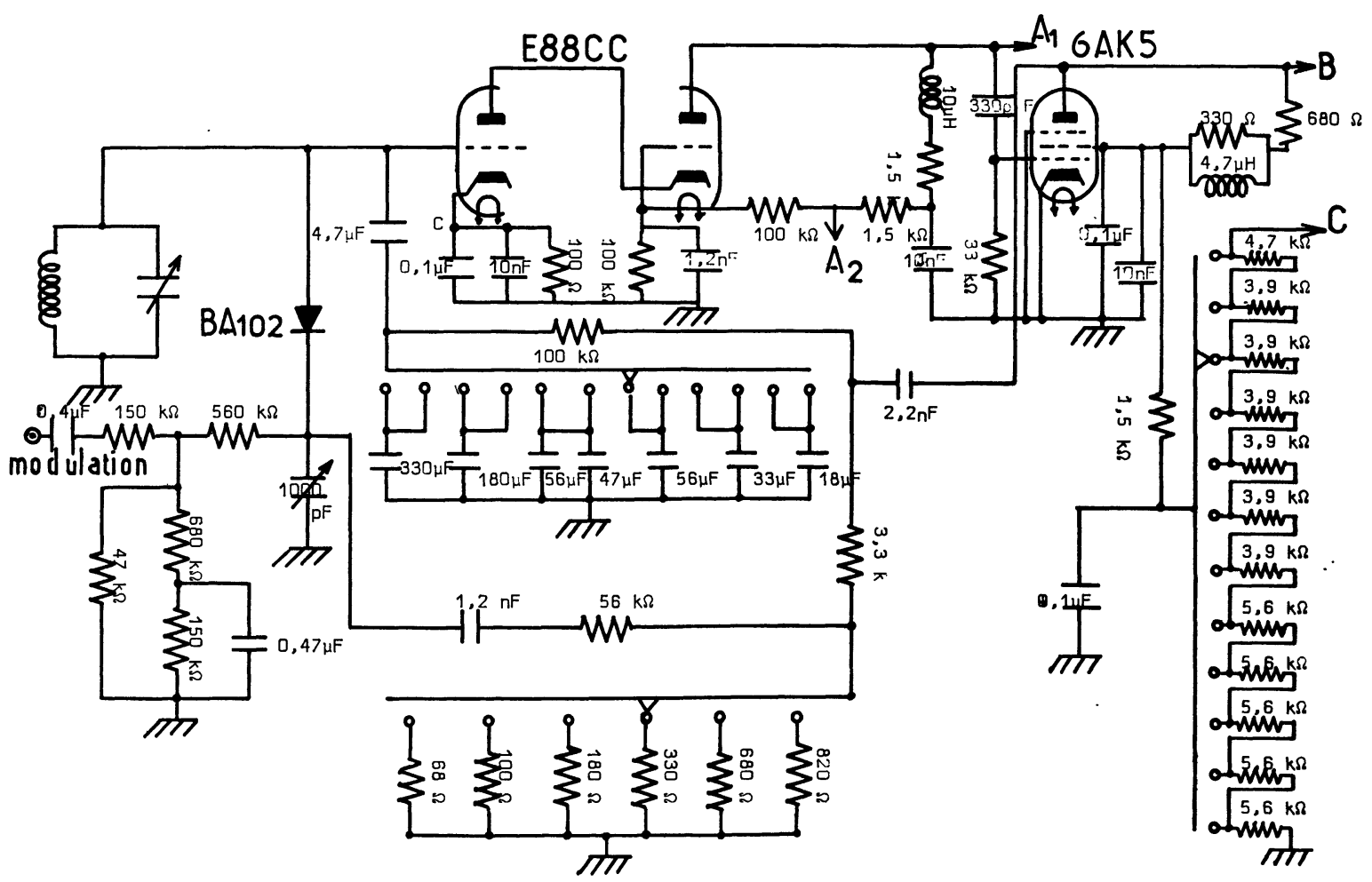

FIg. 1. - Schéma de la tête haute fréquence de l'oscillateur de Robinson. 
avec un étage cascode pour améliorer le facteur de bruit de l'étage amplificateur. L'introduction d'une adaptation d'impédance, dans les circuits de réaction et de compensation, permet d'utiliser des contacteurs miniatures, sans être gêné par les capacités parasites qu'ils introduisent en parallèle sur les résistances de réaction. Cela procure une grande commodité de fonctionnement quand on change les conditions de travail (passage de la température ambiante à celle de l'azote liquide par exemple) $\left(^{1}\right)$.

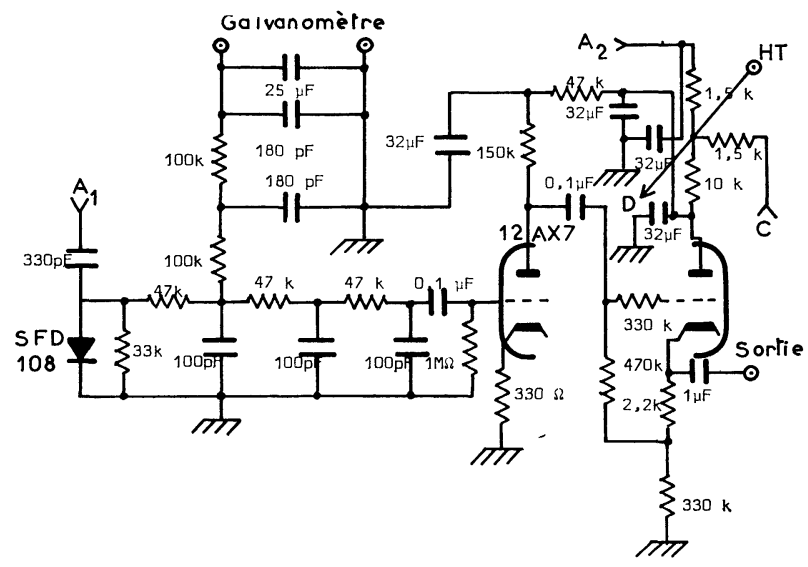

FIG. 2. - Etage préamplificateur basse fréquence.

Un étage d'isolement (Fig. 3), suivi d'un étage à charge cathodique a été prévu pour découpler le compteur de fréquence du spectrographe. Il est, en outre, nécessaire de prendre de grandes précautions dans la réalisation des retours de masse du compteur de

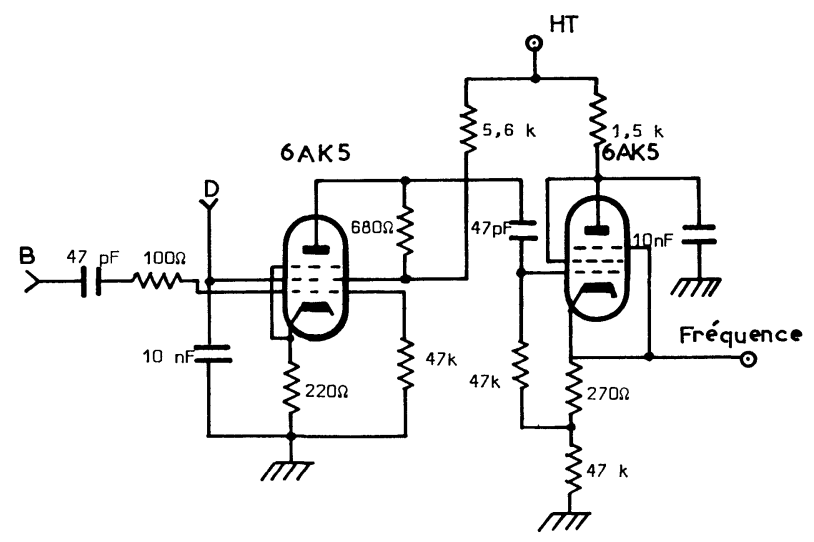

FIG. 3. - Bloc de sortie sur fréquencemètre.

fréquence et des différents châssis du spectrographe, pour éviter l'apparition de parasites dus aux signaux d'ouverture et de fermeture de la porte d'entrée du compteur.

Le repérage des fréquences de kilohertz en kilohertz est assuré sur l'enregistreur à l'aide d'un marqueur commandé par la sortie B. C. D. du compteur [5].

(1) Un étage préamplificateur B. F. (Fig. 2) permet de réduire l'influence des tensions parasites induites dans les retours de masse entre châssis.
La courbe de réponse en fréquence, relevée sur le spectrographe, entre la grille d'entrée du cascode et la plaque de l'étage écrêteur (Fig. 4) montre que le spectrographe peut fonctionner entre 0,1 et $10 \mathrm{MHz}$. Cette conclusion est valable dans le cas d'une modulation

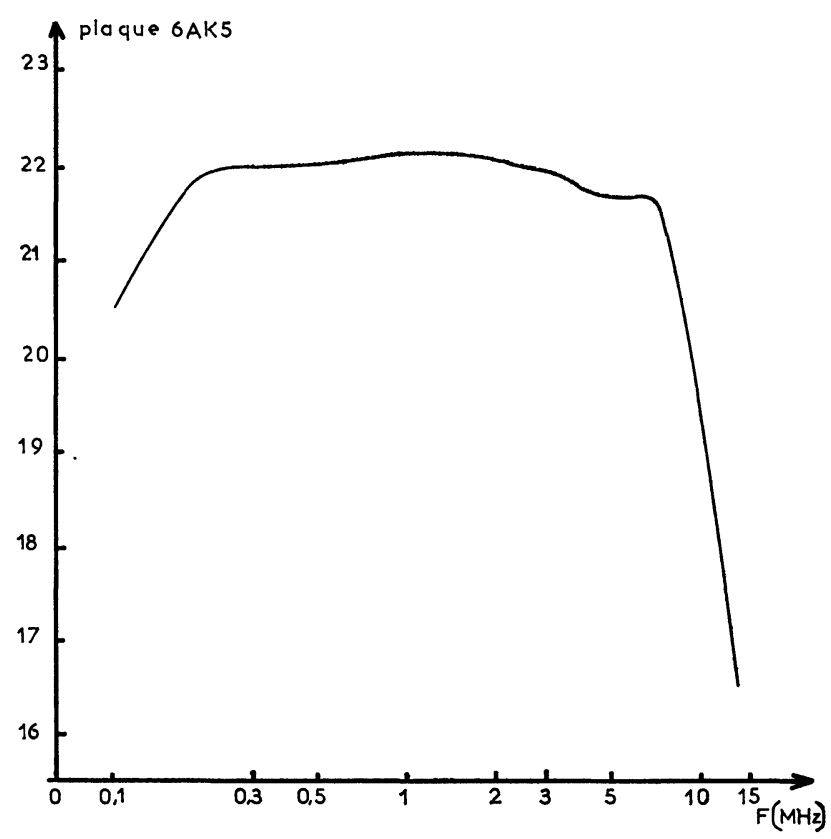

FIG. 4. - Domaine de fonctionnement en fréquence de l'oscillateur H. F. On a utilisé, pour marquer les fréquences, une échelle logarithmique.

magnétique ou modulation Zeeman. Avec une modulation de fréquence, le spectrographe est alors très sensible aux variations du gain avec la fréquence et la gamme utilisable est réduite à $0,5-6 \mathrm{MHz}$ environ.

Les conséquences de cette sensibilité du spectrographe, modulé en fréquence, aux diverses causes de variation du niveau d'oscillation, quand la fréquence varie, sont importantes. Elle explique, bien sûr, l'existence de la modulation d'amplitude parasite et la possibilité de sa correction, suivant la technique utilisée par Robinson [4] $\left({ }^{2}\right)$. Mais, elle explique aussi que cette correction ne soit valable qu'en un point et que le balayage en fréquence s'accompagne d'une dérive de la trace de l'enregistreur. Le sens de cette dérive dépend de l'allure de la réponse globale du spectrographe. Pratiquement, avec les bobines utilisées $(\varphi 3 \mathrm{~cm}$, $L=3$ à $4 \mathrm{~cm}$, diamètre du fil compris entre 0,5 et $1 \mathrm{~mm}$ ) et une capacité d'accord variant entre 75 et $200 \mu \mathrm{F}$, la dérive s'annule au milieu de la gamme couverte par chacune des bobines. Notons aussi que pour les fréquences élevées d'une bobine, la surtension décroît assez vite avec la fréquence pour avoir un effet

(2) Incidemment, notons que cette correction ne peut être totale lorsque le circuit oscillant a une surtension appréciable : il existe un retard entre la correction appliquée (tension sur la varicap) et l'effet de la correction. Un tel retard dans des circonstances semblables a déjà été signalé par Watkins (6). 
analogue à celui de la compensation. Cette dernière peut ne plus être possible avec le dispositif mentionné et il faut recourir à une compensation de la tension parasite dans les étages basse fréquence du spectrographe.

Le même phénomène se produit aussi à l'extrémité haute fréquence de la réponse du spectrographe.

Enfin, la sensibilité du spectrographe à la réponse en fréquence du niveau d'oscillation explique les signaux parasites obtenus en modulation de fréquence avec les échantillons piézoélectriques.

L'importance de l'effet des variations de surtension sur la modulation d'amplitude parasite et sur la dérive, nous a conduits à étudier la surtension propre des " varicaps » utilisées pour la modulation de fréquence en vue de leur utilisation éventuelle pour des balayages d'amplitude limitée à quelques dizaines de kilohertz.

Nous avons ainsi étudié une varicap BA 102, un varactor MV 1738, ainsi que des transistors planars épitaxiaux (montés en diode avec la base reliée à l'émetteur) et un transistor à alliage. Les courbes de la variation de capacité avec la polarisation, et de la variation correspondante de surtension (Fig. 5 et 6 ) montrent que la variation de capacité des transistors

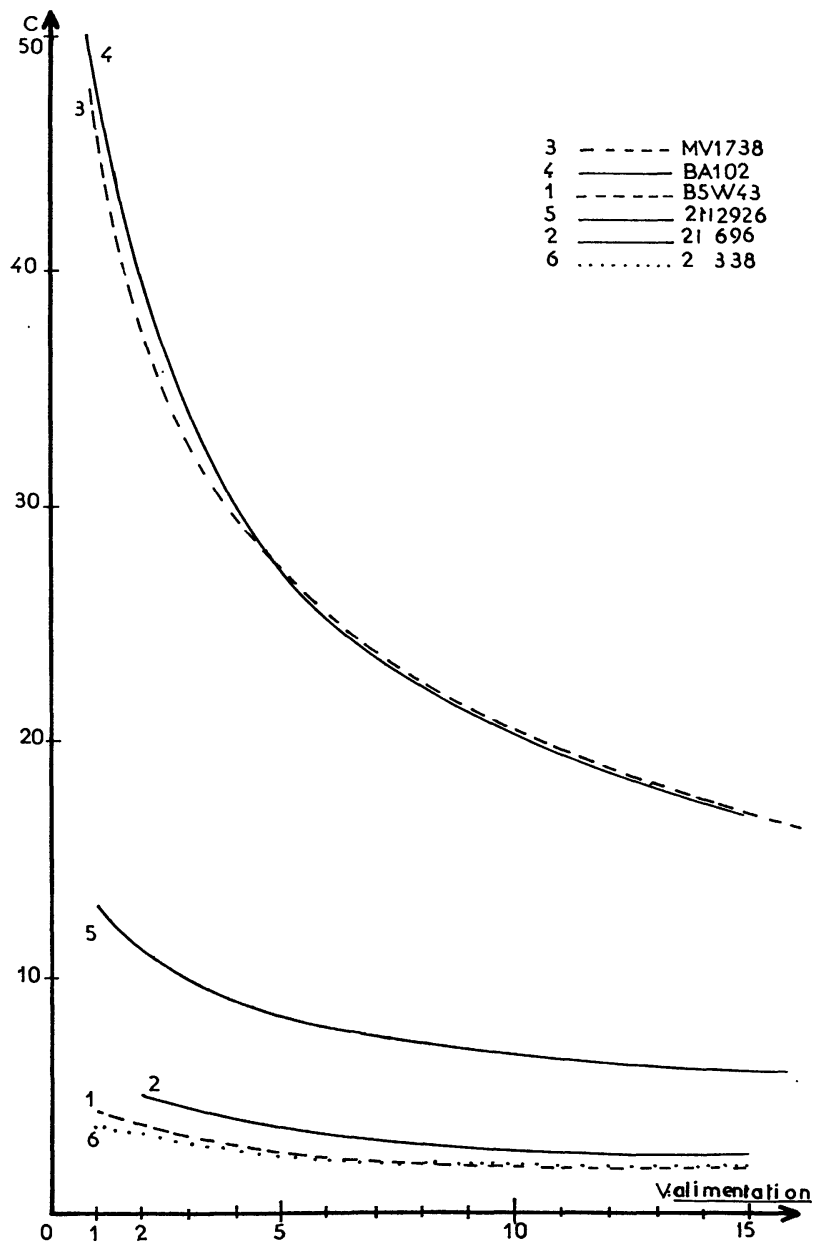

Fig. 5. - Graphe de variation de la capacité de divers composants pouvant servir de modulateurs de fréquence, en fonction de la tension continue appliquée.

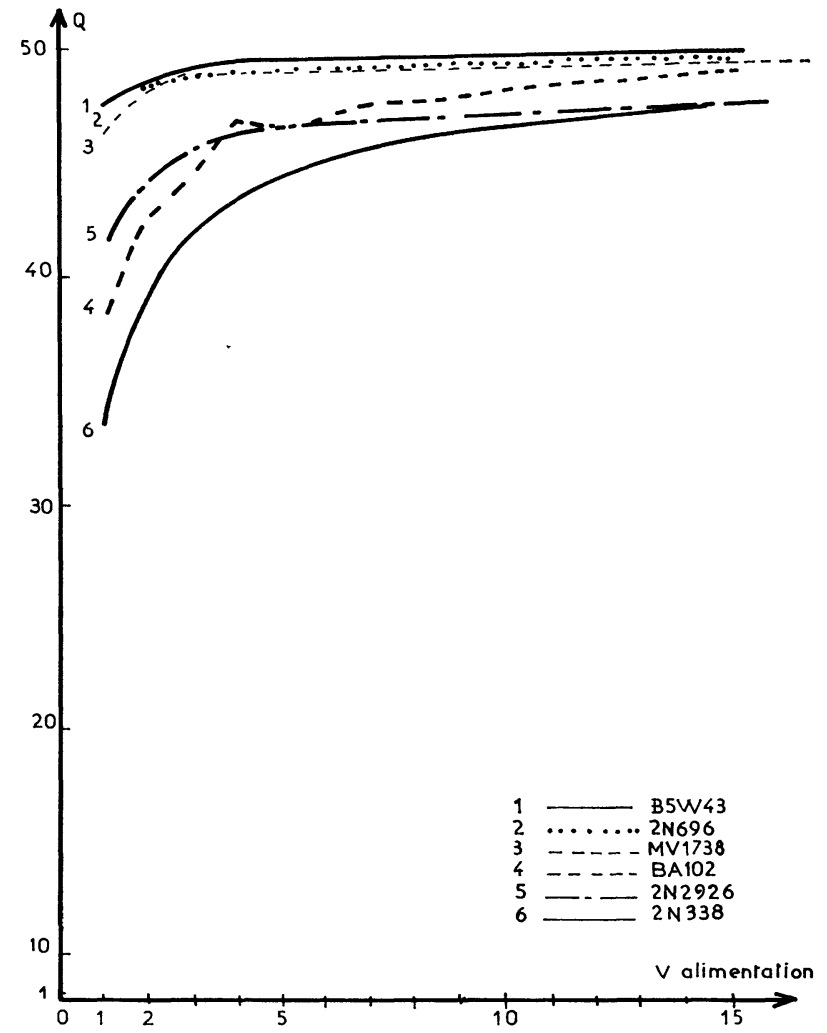

Fig. 6. - Etude du facteur de qualité de divers composants pouvant servir de modulateurs de fréquence, en fonction de la tension continue appliquée.

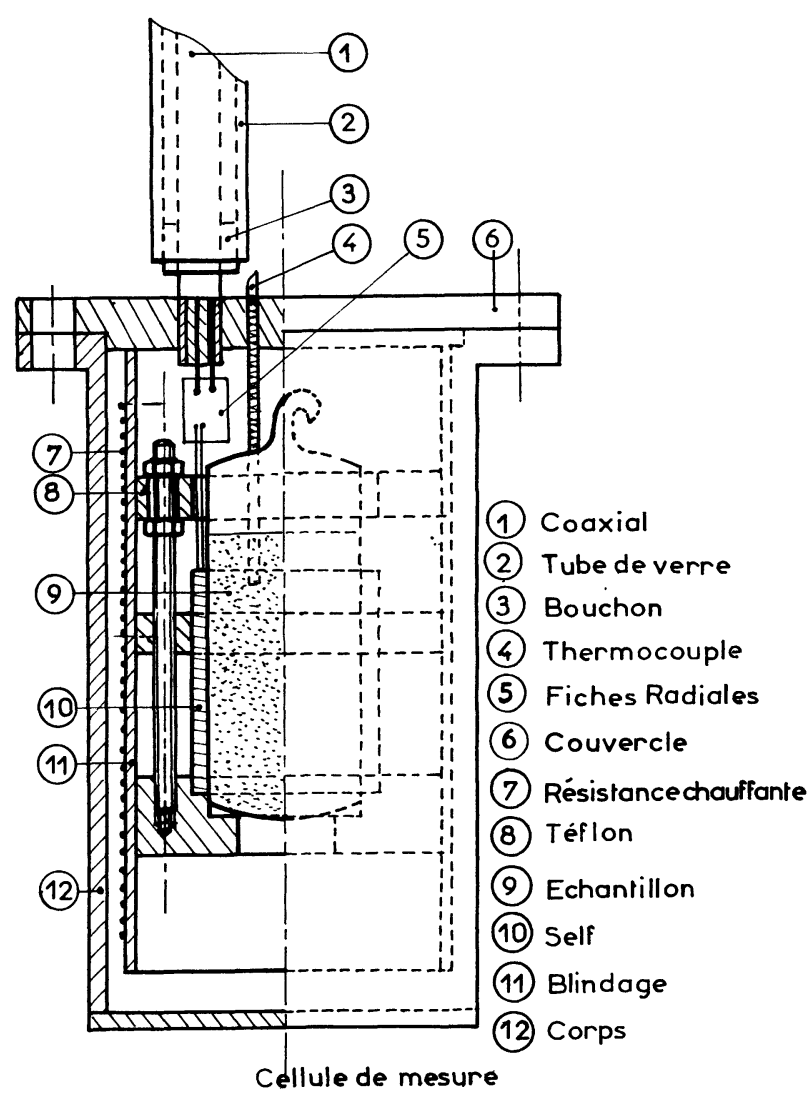

FIG. 7. - Cellule de mesure. 
montés en diode est peu importante et inutilisable pratiquement, et que la diode BA 102 est nettement moins bonne que le varactor MV 1738.

1.2 LA Cellule De MESURE. - Son fonctionnement est basé sur le principe de l'inertie thermique d'une masse métallique importante, à savoir le corps de la cellule (Fig. 7).

La variation de la température s'effectue, à partir de la température de l'azote liquide, au moyen d'une résistance chauffante (7) enroulée autant de fois dans un sens que dans l'autre afin d'éviter toute création de champ magnétique supplémentaire, autour d'un blindage (11) entourant la bobine (10). La réalisation d'un blindage de diamètre élevé $(68 \mathrm{~mm})$, comparé à celui des bobines $(32 \mathrm{~mm})$, permet de ne pas atténuer de plus de $5 \%$ le facteur de qualité $Q$ de la self.

La mesure de la température s'effectue à l'aide d'un thermocouple (4), type chromel-alumel, et d'un millivolmètre èlectronique PM 2440.

2. Résultats expérimentaux. -2.1 RECHERCHE ET OBSERVATION DE RAIES. - Nous avons, dans une première étape, recherché et observé des raies (Tableau I) dans trois composés : orthophénylènediamine, 2 méthyl-pyridine et cyanure d'allyle. Les couplages obtenus pour les deux premiers composés sont en accord avec ceux de travaux récents [7], [8], [9].

Par contre, aucun signal n'a été observé dans les molécules d'intérêt biologique suivantes: guanine, thymine et uracile. Il en a été de même pour des acides aminés : glycine, L. thyrosine et D. L. méthionine.

Pour ces trois derniers composés, l'inobservation des résonances est vraisemblablement due à un couplage trop faible : c'est le cas de la glycine pour laquelle le couplage a pu être déterminé par R. M. N. [10] : il est de l'ordre du mégahertz et la fréquence correspondante serait de 0,7 MHz. Une recherche à cette fréquence n'a, par ailleurs, pas permis d'observer la résonance, la relaxation est, ainsi qu'on a pu s'en rendre compte par R. M. N. à température ambiante, très faible.

Pour les composés hétérocycliques, il est vraisemblable que le couplage des atomes d'azote y est, du moins pour certains d'entre eux, de l'ordre de grandeur de ceux rencontrés pour les azabenzènes [7] et l'absence de signal doit être attribuée à un faible coefficient de remplissage d'une part, et à un temps de relaxation long d'autre part.

2.2 DisCusSION DES COUPlages OBSERVÉS. - 2-picoline. - Le couplage et l'asymétrie $\left(e^{2} Q q_{\text {moy }}=4,480\right.$; $\left.\eta_{\text {moy }}=0,335\right)$ ne diffèrent pas de ceux de la 4-picoline $\left(e^{2} Q q_{\text {moy }}=4,414 ; \eta=0,342\right)$ de quantités supérieures à l'ordre de grandeur des effets intermoléculaires et on ne peut que constater, comme pour la 4-picoline, les variations de couplage et d'asymétrie par rapport à la pyridine $\left(e^{2} Q q_{\text {moy }}=4,584 ; \eta_{\text {moy }}=0,396\right)$; variations qui ont été interprétées à partir de l'effet donneur d'électron du substituant méthyl [11].

Cyanure d'allyle. - C'est avec l'acylonitrile et le méthacylonitrile [12] un des rares nitriles éthyléniques dont la résonance de l'azote ait été observée. La différence (Tableau II) entre le cyanure d'allyle et les deux autres nitriles cités est essentiellement due à l'éloignement de la double liaison du groupe cyanure. Il semble que cela doive empêcher une conjugaison entre la double liaison éthylénique et une des doubles liaisons $\mathrm{du}$ groupe nitrile. Pratiquement, si on se reporte au tableau I on ne voit pas de différences notables entre les couplages ou les asymétries et il y a lieu de penser qu'il n'y a pas d'interaction du groupe nitrile avec la double liaison de la chaîne.

\section{TABleaU I}

Résonances observées:

Orthophénylènediamine.

$$
\begin{aligned}
& v_{1}=3,1502 \mathrm{MHz} \\
& v_{1}^{\prime}=3,1308 \mathrm{MHz} \\
& v_{2}=2,5386 \mathrm{MHz} \\
& v_{2}^{\prime}=2,4977 \mathrm{MHz}
\end{aligned}
$$

$$
\begin{aligned}
v_{1_{\text {moy }}} & =3,1405 \mathrm{MHz} \\
v_{2_{\text {moy }}} & =2,5181 \mathrm{MHz} \\
e^{2} Q q & =4,5019 \mathrm{MHz} \\
\eta \quad & 0,3391 \\
e^{2} Q q & =4,4593 \\
\eta \quad & =0,3309
\end{aligned}
$$$$
e^{2} Q q_{\text {moy }}=3,772 \mathrm{MHz}
$$$$
\eta_{\text {moy }}=0,330
$$

2-picoline.

$$
\begin{aligned}
& v_{1}=3,7583 \mathrm{MHz} \\
& v_{2}=2,9945 \mathrm{MHz} \\
& v_{1}^{\prime}=3,7135 \mathrm{MHz} \\
& v_{2}^{\prime}=2,9759 \mathrm{MHz}
\end{aligned}
$$

$$
\begin{aligned}
e^{2} Q q_{\text {moy }} & =4,481 \mathrm{MHz} \\
\eta_{\text {moy }} & =0,335
\end{aligned}
$$

\section{Cyanure d'allyle.}

$$
\begin{aligned}
& v_{1}=2,9475 \mathrm{MHz} \\
& v_{2}=2,8287 \mathrm{MHz}
\end{aligned}
$$$$
\begin{aligned}
e^{2} Q q & =3,8508 \\
\eta & =0,0617
\end{aligned}
$$ 


\section{TABLEAU II}

Couplages dans quelques nitriles éthyléniques et dans un nitrile saturé [7].

Acylonitrile

Méthacylonitrile

Cyanure d'allyle

Butyronitrile

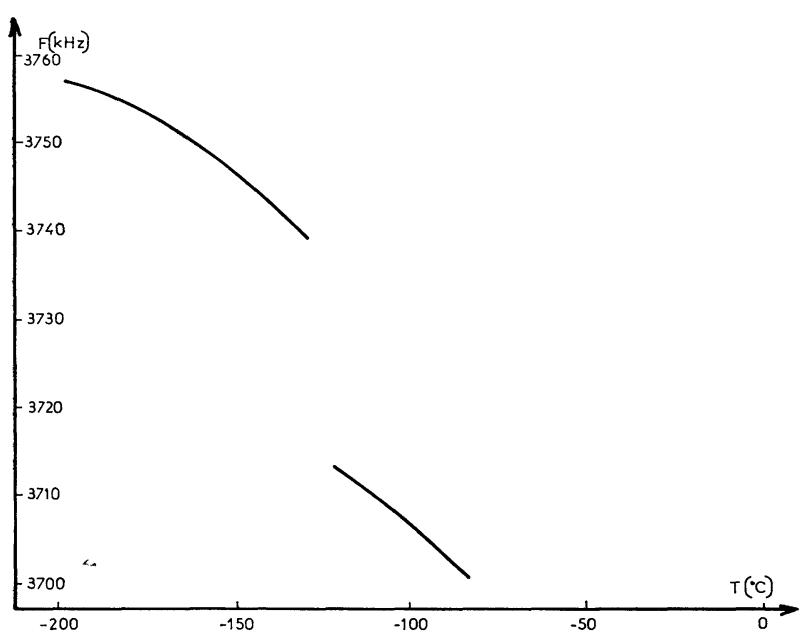

FIG. 8. - Transition de phase de la 2-picoline.

Orthophénylènediamine. - Les valeurs du couplage et de l'asymétrie peuvent être comparées à celles des composés voisins : aniline et paraphénylènediamine comme l'indique le tableau III.

\section{TABLEAU III}

Couplage dans les amines aromatiques.

$$
\begin{aligned}
& \text { Aniline } \\
& e^{2} Q q_{\text {moy }}=3,933 \mathrm{MHz} \\
& e^{2} Q q_{\mathrm{moy}}=3,910 \mathrm{MHz} \\
& \epsilon^{2} Q q_{\mathrm{moy}}=3,772 \mathrm{MHz} \\
& \eta_{\text {moy }}=0,269 \\
& \text { (8-14) } \\
& \eta_{\text {moy }}=0,330
\end{aligned}
$$

Dans ces deux composés, les couplages et asymétries quasi identiques révèlent que les deux groupes $\mathrm{NH}_{2}$ de la paraphénylènediamine sont pratiquement indépendants. Dans le cas de l'orthophénylènediamine, on peut penser que la participation des électrons « $\mathrm{n}$ » de l'azote au système « $\pi$ » du cycle benzénique est facilitée par l'établissement d'une structure diénique.

$$
\mathrm{N}=\mathrm{C}-\mathrm{C}=\mathrm{N} \text {. }
$$

Une telle structure réduisant la population du doublet libre de l'atome d'azote expliquerait une diminution du couplage et une augmentation de l'asymétrie. Ce schéma est très simplifié car il ne tient pas compte d'un changement d'hybridation accompagnant l'établissement de la structure diénique proposée : il est vraisemblable que les groupes $-\mathrm{NH}_{2}$ doivent avoir une structure plus proche de la structure plane dans l'orthophénylènediamine que dans l'aniline ou la paraphénylènediamine. Un tel modèle permet de comprendre, suivant la méthode habituelle de calcul des composants du gradient de champ, que la variation d'asymétrie « $\eta$ » soit beaucoup plus importante que la variation de couplage $« e^{2} Q q »$.

2.3 INFLUENCE DE LA TEMPÉRATURE SUR LA FRÉQUENCE DE RÉSONANCE. - La dépendance de la fréquence de résonance avec la température a été relevée pour la 2-picoline et l'orthophénylènediamine. Une transition de phase réversible a été obtenue pour la 2-picoline. Cette transition avait été détectée préalablement par l'observation de deux résonances différentes, à la température de l'azote liquide, suivant la rapidité de refroidissement de l'échantillon. Il s'agit d'une transition du $1^{\text {er }}$ ordre avec un changement de structure expliquant la variation de fréquence de $25 \mathrm{kHz}$ observée vers $-130^{\circ} \mathrm{C}$ sur la raie $v_{1}$.

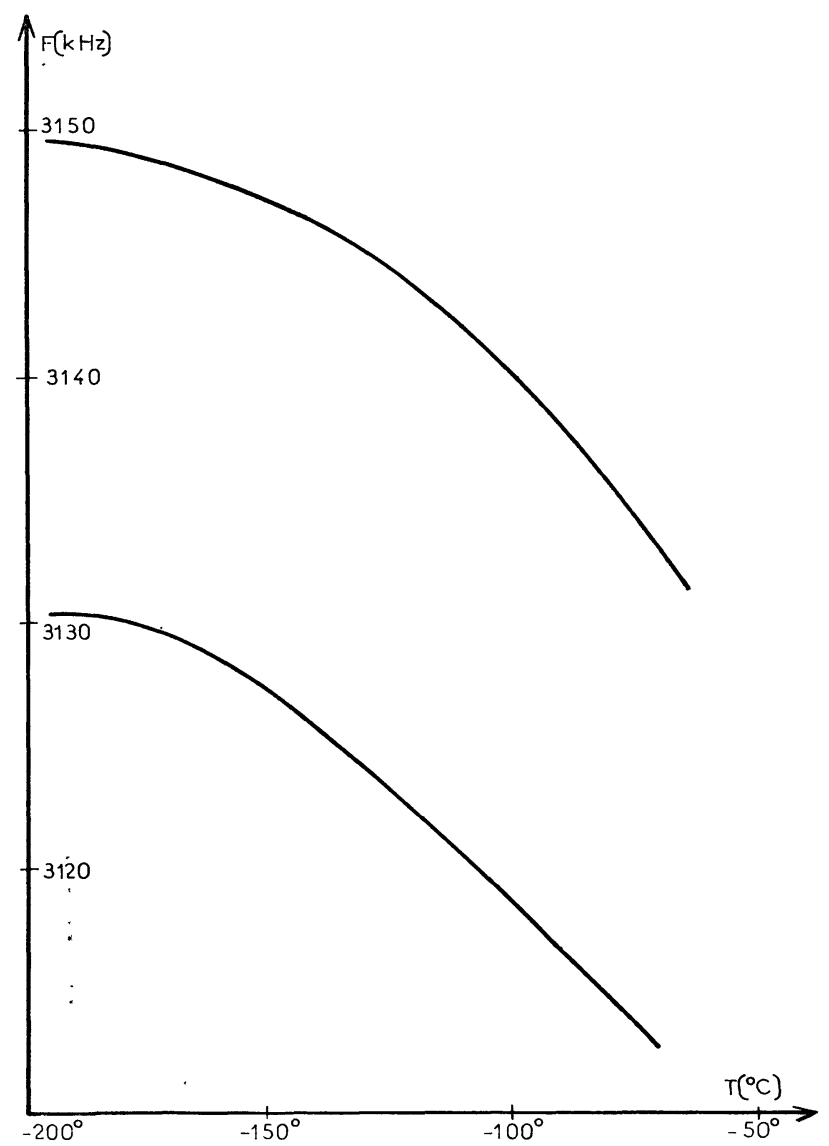

Fig. 9. - Etude du glissement de fréquence pour deux raies de l'orthophénylènediamine, en fonction de la température.

L'interprétation des courbes obtenues, en terme de mouvements moléculaires, n'est pas en général possible car les équations d'évolution [13] du couplage 
font intervenir trois mouvements et on ne dispose que de deux raies $v_{1}$ et $v_{2}$ pour les déterminer. Une étude de relaxation donnerait une relation supplémentaire par la comparaison des temps $T_{1}$ pour les deux raies $v_{+}$ et $v_{-}$[13]. En outre, dans les deux composés étudiés, l'absence de symétrie dans la répartition des groupes complique l'expression donnée par Barton [13] puisque les axes d'inertie ne sont pas confondus avec les axes principaux des tenseurs gradients de champ électrique.

Dans le cas de l'orthophénylènediamine, nos résultats sont analogues à ceux obtenus par Yim et Coll. [9].

\section{Bibliographie}

[1] Mallet (G.), Thèse $3^{\mathrm{e}}$ cycle, Marseille, 1969.

[2] Guibe (L.), non publié.

[3] Robinson (F. N. H.), J. Sci. Instrum., 1959, 36, 481.

[4] Robinson (F. N. H.), Rev. Sci. Instrum., 1963, 34, 1260.

[5] Guibe (L.) et Berteloot (M.), J. Sci. Instrum., 1958, série 2, 1950.

[6] Watkins (G. D.), Thèse Harvard, 1952.

[7] Guibe (L.) et LUCKEN (E. A. C.), Molecular Spectr., $1968,14,79$.

[8] Ikeda (R.), ONDA (S.), Nokamura (D.) et Rubo (M.), J. Phys., Chim. 1968, 72, 2501.
[9] Yim (C. T.), Whitehead (M. A.) et Lo (D. H.), Can.J. Chem., 1968, 46, 3595.

[10] ANdersson (L. O.), Gourdji (M.), Guibe (L.) et Proctor (W. G.), C. R. Acad. Sci., Paris, 1968, 267, 803.

[11] LuCken (E. A. C.), Trans. Faraday Soc., 1969, 57, 729.

[12] Colligiani (A.), Guibe (L.), Haigt (P. J.) et LuCKen (E. A. C.), Molecular Phys., 1968, 14, 73.

[13] Barton (B. L.), J. Chem. Phys., 1967, 46, 1553.

[14] Eletr (S.), Thèse, Berkeley, 1968.

[15] Minematsu (M.), J. Phys. Soc. Japan, 1959, 14, 1030. 